\title{
Single-Atom Absorption Imaging
}

\author{
E. W. Streed, A. Jechow, B. G. Norton, M. J. Petrasiunas, D. Kielpinski \\ Centre for Quantum Dynamics, Griffith University, 170 Kessels Road Brisbane, Australia 4111 \\ Presenting author e-mail address: e.streed@griffith.edu.au
}

\begin{abstract}
We demonstrate the first absorption imaging of a single atom. Absorption depths of up to $2.6(2) \%$ were observed for laser cooled ${ }^{174} \mathrm{Yb}^{+}$ions illuminated at $369.5 \mathrm{~nm}$ and imaged with a phase Fresnel lens.
\end{abstract}

\section{Introduction}

Absorption is a key process in the interaction of electromagnetic fields with matter. The simplest instance of this is the absorption of a photon by an allowed atomic transition. While absorption imaging is often used to great effect in optically dense ensembles such as Bose-Einstein condensates, the small cross section of atomic transitions makes recovery of an absorption signal from a single atom with standard low-numerical-aperture optics [1] challenging at best. The recent application of high numerical aperture optics with near diffraction-limited performance to atomic systems [2-4] provides the capability to obtain substantial absorption effects and create strong atom-photon interactions. The latter is a necessary component for creating efficient coupling between photons and atoms in scalable quantum information processing systems. While imaging of the absorption induced by a single molecule has recently been demonstrated [5], no previous work has imaged the absorption from a single atom.

\section{Apparatus}

A double-needle RF Paul trap was used to confine ${ }^{174} \mathrm{Yb}^{+}$ions loaded by isotope selective photoionisation from an atomic beam. The ion was laser cooled on the $369.5 \mathrm{~nm} \mathrm{~S}_{1 / 2}-\mathrm{P}_{1 / 2}$ transition using light from an ultraviolet external cavity laser diode (ECLD) that was frequency stablised to ions in an electrical discharge [6]. Decay from the $\mathrm{P}_{1 / 2}$ state into the meta-stable ${ }^{2} \mathrm{D}_{3 / 2}$ state $(0.5 \%$ probability) interrupted the scattering of $369.5 \mathrm{~nm}$ light unless repumped by driving the $935.2 \mathrm{~nm}$ transition with another ECLD. To obtain trapping times of several hours light from a 638.2 $\mathrm{nm}$ ECLD was used to repump the atom from infrequent decays into the meta-stable $\mathrm{F}_{7 / 2}$ state induced by collisions with the background gas. Additional details of the trapping and laser systems have been previously documented $[3,6,7]$.

The ion was illuminated for absorption imaging with $369.5 \mathrm{~nm}$ cooling light focused to a few-micron-diameter spot with an in-vacuum aspheric lens (Thorlabs 352671-405, $\mathrm{f}=4.02 \mathrm{~mm}$ ). The light was then recollimated by a phase Fresnel lens $(0.64$ numerical aperture, $\mathrm{f}=3 \mathrm{~mm})$ and reimaging onto a cooled CCD camera with $615 \mathrm{x}$ magnification. In fluorescence imaging this optical configuration has demonstrated spot sizes down to $440 \mathrm{~nm}$ FWHM [3]. To obtain reference images $935.2 \mathrm{~nm}$ repumping beam was blocked and the ion shelved into the metastable $\mathrm{D}_{3 / 2}$ state, in which the ion does not scatter $369.5 \mathrm{~nm}$ light. The $369.5 \mathrm{~nm}$ illumination beam was linearly polarized to prevent optical pumping and the power stablised minimize fluctuations between reference and signal images.

\section{Results}

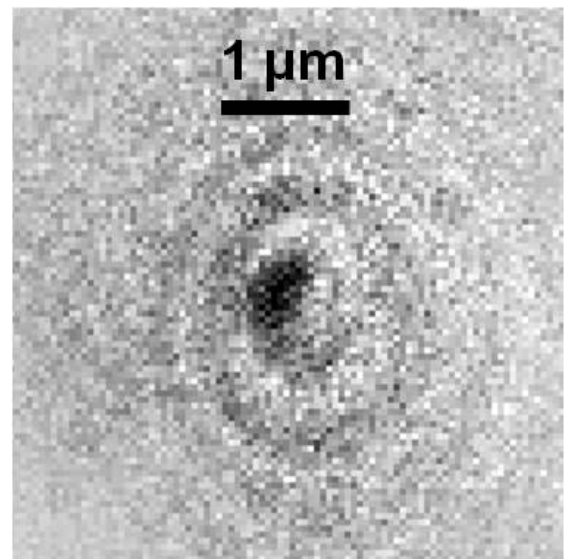

Figure 1. Absorption image of a single ${ }^{174} \mathrm{Yb}^{+}$atom in vacuum. 
Images of the $369.5 \mathrm{~nm}$ illumination beam with and without the ion scattering were subtracted and an image of the absorption by a single ${ }^{174} \mathrm{Yb}^{+}$atom was obtained. Figure 1 illustrates a typical absorption image. Reference and signal images were taken within a few seconds of each other to minimize differences in the images arising from slight mechanical drift in the system. Variations between the two result in small offsets across the image. Image acquisition times were much shorter than for fluorescence imaging to prevent the high signal level from saturating the camera. The absorption spot sizes were observed to be similar to those obtained from fluorescence imaging [3] under optimum conditions.

The dependence of the absorption depth on illumination laser detuning and power were investigated (Figure 2) and found to be generally consistent with previous results for fluorescence imaging [3,6,7]. The effects of recoil heating were pronounced for detunings less then the half linewidth. At these detunings, excess motion of the ion due to recoil heating blurred the ion image and reduced the absorption depth. The point at the extreme right of Figure $2 \mathrm{~A}$ was taken with a detuning slightly blue of resonance and exhibits an absorption depth close to zero due to the large spatial extent of the hot ion. The maximum absorption of $2.6(2) \%$ was observed at a detuning of $-8 \mathrm{MHz}$, half the linewidth to the red of the heat/cool point. This corresponded to the detuning of the half the maximum fluorescence. The maximum absorption without this recoil heating effect would be expected to be double that observed. From this we estimate a maximum absorption depth of 5.2(4)\% would be expected without the limitations presented by the laser cooing dynamics. The absorption signal at a constant detuning of $-8 \mathrm{MHz}$ was likewise observed to saturate at high illumination intensities (Figure 2B).
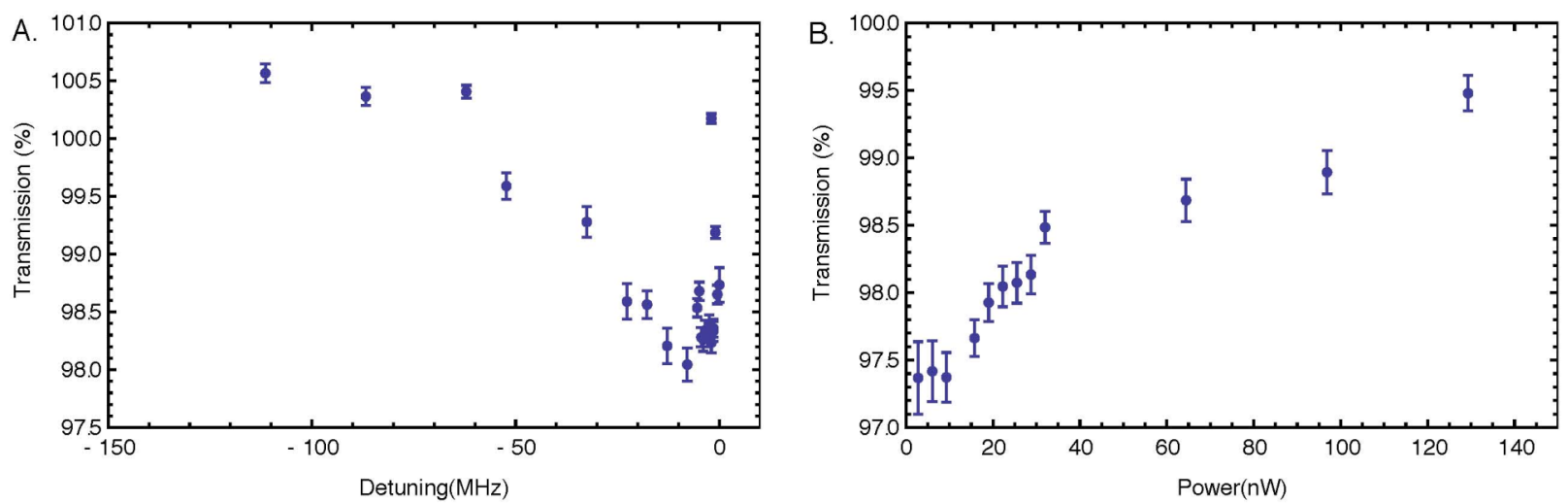

Figure 2. Dependence of absorption on laser detuning from resonance (A) at fixed power of $15 \mathrm{nW}$ and on optical power at fixed detuning of $-8 \mathrm{MHz}(\mathrm{B})$.

\section{Acknowledgements}

This work is funded by the Australian Research Council under DP0773354 (D. K.), DP0877936 (E. S., Australian Postdoctoral Fellowship), and FF0458313 (H. Wiseman, Federation Fellowship), as well as the U.S. Air Force Office of Scientific Research (USAFOSR) (FA2386-09-1-4015). A. J. is supported by a Griffith University Postdoctoral Fellowship. The PFL was fabricated by Margit Ferstl of the Heinrich-Hertz-Institut of the FraunhoferInstitut fur Nachrichtentechnik in Germany.

\section{References}

[1] D. J. Wineland, W. M. Itano, and J. C. Bergquist, “Absorption spectroscopy at the limit: detection of a single atom.” Opt. Lett. 12, 389-391 (1987).

[2] Tey, M. K. et al. "Strong interaction between light and a single trapped atom without the need for a cavity." Nat. Phys. 4, $924-927$ (2008).

[3] A. Jechow, E. W. Streed, B. G. Norton, M. J. Petrasiunas, and D. Kielpinski, "Wavelength-scale imaging of trapped ions using a phase Fresnel lens." Opt. Lett. 36 1371-1373 (2011)

[4] N. Piro, F. Rohde, C. Schuck, M. Almendros, J. Huwer, J. Ghosh, A. Haase, M. Hennrich, F. Dubin, and J. Eschner, "Heralded single-photon absorption by a single atom," Nat. Phys. 7, 17 (2010).

[5] M. Celebrano, P. Kukura, A. Renn, and V. Sandoghdar, "Single-molecule imaging by optical absorption." Nat. Phot. 5, 95-98 (2011).

[6] E.W. Streed, T.J. Weinhold, and D. Kielpinski, "Frequency stabilization of an ultraviolet laser to ions in a discharge," Appl. Phys. Lett. 93, 071103 (2008).

[7] E. W. Streed, B. G. Norton, A. Jechow, T. J. Weinhold, and D. Kielpinski, "Imaging of Trapped Ions with a Microfabricated Optic for Quantum Information Processing.” Phys. Rev. Lett. 106, 010502 (2011). 